\title{
Cutaneous Dengue Virus Inoculation Triggers Strong B Cell Reactions but Contrastingly Poor T Cell Responses
}

\author{
Edith Marcial-Juárez ${ }^{1}$ (D) Julio García-Cordero ${ }^{2} \cdot$ Raúl Antonio Maqueda-Alfaro $^{1} \cdot$ Rafael Eduardo Saucedo-López $^{1}$ • \\ Luvia Enid Sánchez-Torres ${ }^{3} \cdot$ Leticia Cedillo-Barrón $^{2} \cdot$ Leopoldo Flores-Romo $^{1}$ (])
}

Received: 16 October 2019/Accepted: 24 December 2019/Published online: 20 April 2020

(C) Wuhan Institute of Virology, CAS 2020

\begin{abstract}
Dengue is a global health problem without current specific treatment nor safe vaccines available. While severe dengue is related to pre-existing non-neutralizing dengue virus (DENV) antibodies, the role of $\mathrm{T}$ cells in protection or pathology is unclear. Using cutaneous DENV infection in immunocompetent mice we previously showed the generation of PNA+ germinal centers (GCs), now we assessed the activation and proliferation of B and T cells in draining lymph nodes (DLNs). We found a drastic remodelling of DLN compartments from 7 to 14 days post-infection (dpi) with greatly enlarged B cell follicles, occupying almost half of the DLN area compared to $\sim 24 \%$ in naïve conditions. Enormous clusters of proliferating (Ki-67+) cells inside B follicles were found 14 dpi, representing 33\% of B cells in DLNs but only 2\% in noninfected mice. Inside GCs, we noticed an important recruitment of tingle body macrophages removing apoptotic cells. In contrast, the percentage of paracortex area and total $\mathrm{T}$ cells decreased by 14-16 dpi, compared to controls. Scattered randomly distributed Ki-67+ T cells were found, similar to non-infected mice. CD69 expression by CD4+ and CD8+ T cells was minor, while it was remarkable in B cells, representing $1764.7 \%$ of change from basal levels 3 dpi. The apparent lack of $\mathrm{T}$ cell responses cannot be attributed to apoptosis since no significant differences were observed compared to noninfected mice. This study shows massive B cell activation and proliferation in DLNs upon DENV infection. In contrast, we found very poor, almost absent CD4+ and CD8+ T cell responses.
\end{abstract}

Keywords Dengue virus (DENV) $\cdot$ B and T cells proliferation $\cdot$ Lymphocyte activation $\cdot$ Lymph nodes $\cdot$ In vivo cutaneous infection · Immunocompetent mice

This article is dedicated to the memory of Professor Leopoldo Flores-Romo, a brilliant Mexican immunologist and an inspiring scientist mentor.

Leopoldo Flores-Romo-deceased.

Edith Marcial-Juárez

edith.mjuarez@gmail.com

$\triangle$ Leopoldo Flores-Romo

lefloresromo@gmail.com

1 Department of Cell Biology, Center for Advanced Research (CINVESTAV-IPN), The National Polytechnic Institute, 07360 Mexico City, Mexico

2 Department of Molecular Biomedicine, Center for Advanced Research (CINVESTAV-IPN), The National Polytechnic Institute, 07360 Mexico City, Mexico

3 Immunology Department, The National School of Biological Sciences (ENCB-IPN), The National Polytechnic Institute, 11340 Mexico City, Mexico

\section{Introduction}

Dengue is a worldwide viral disease manifested as several clinical entities, from an asymptomatic form to acute selflimiting dengue fever (DF), to a life-threatening haemorrhagic disease, severe dengue (SD) (WHO 2019). Dengue virus (DENV 1-4) is transmitted among humans by a female Aedes aegypty mosquito bite. Because of the vector distribution around the globe, more than half of the world population is at risk, with an estimated of 96 million clinical cases annually and around $2.5 \%$ of hospitalized cases ending in fatalities (Bhatt et al. 2013; WHO 2019).

Since the first recognized DENV epidemics in the 1780's (Rush 1789; Dick et al. 2012), the study of DENV has faced enormous challenges, such as the broad range of clinical presentations, the co-circulation of four DENV serotypes in endemic areas plus the recently emerging related flaviviruses (e.g. Zika virus), the severe pathology 
during heterologous infection and the lack of an ideal animal model to investigate the disease. In addition, urbanization, poor vector control, climate change and viral and vector evolution have contributed to the 30-fold increase in global incidence over the past 50 years (WHO 2012).

In severe cases, usually during heterologous secondary infections, haemorrhagic manifestations such as vascular permeability, bleeding, plasma leakage and thrombocytopenia start once the fever/viremia remits (5-7 days after first symptoms), suggesting that the pathology might be related more with the adaptative immune response than with the viral replication (Mongkolsapaya et al. 2006). Therefore, a better understanding of the immune responses triggered by DENV in primary and secondary infections is urgently needed for a more rational design of antiviral drugs and especially safe vaccines (Arkin 2019; Chatterjee 2019).

Whereas the role of protective neutralizing antibodies in the control of homotypic DENV reinfections has been established (Sabin 1952; Buddhari et al. 2014), cross-reactive non-neutralizing heterotypic antibodies have been related to infection facilitation (Sangkawibha et al. 1984; Burke et al. 1988; Rothman 2004). Cells bearing Fc $\gamma$ receptors will uptake opsonized viral particles in a phenomenon called antibody-dependent enhancement (ADE), thus contributing to the immunopathology (Halstead 1970; Halstead and O'Rourke 1977). The mechanisms generating antibodies of different affinities to DENV remain to be elucidated, especially regarding the $\mathrm{B}$ cell differentiation into antibody secreting-plasma cells in lymphoid organs, either through germinal center (GC) reactions or by extrafollicular responses.

For some viruses such as human immunodeficiency virus, respiratory syncytial virus, influenza A virus or coronavirus, it is well known the crucial involvement of $\mathrm{T}$ cells for viral clearance (Yap et al. 1978; Taylor and Askonas 1986; Cannon et al. 1987; Slütter et al. 2013; Channappanavar et al. 2014; Ndhlovu et al. 2015). This occurs by various mechanisms such as cytokine production, cytotoxic activity, CD8 $+\mathrm{T}$ cell degranulation, and enhancement of $\mathrm{B}$ cell responses. In contrast, the role of $\mathrm{T}$ cells in the resolution, control, protection and/or in the immunopathology of DENV infection is poorly understood (Malavige and Ogg 2013; Rivino 2016).

Using an in vivo model of DENV infection in immunocompetent mice, we previously showed the generation of PNA $+\mathrm{GCs}$, the expression of structural (E and PreM) and non-structural (NS3) DENV proteins inside draining lymph nodes (DLNs) and the production of DENV specific antibodies upon cutaneous DENV-2 inoculation (Yam-Puc et al. 2015). Now, we have assessed in more detail the potential remodeling of the main DLN compartments, we also studied the proliferation and activation on B cells and extended this to the parallel studies of $\mathrm{T}$ cells. We found very striking differences between $\mathrm{T}$ and $B$ cell responses after DENV infection, with a massive early activation and vigorous proliferation of $\mathrm{B}$ cells followed by a significant recruitment of Tingle Body Macrophages (TBMs) inside GCs. Simultaneously, we observed a contrastingly poor, almost absent $\mathrm{T}$ cell response during the course of infection.

\section{Matherials and Methods}

\section{Animals and Immunizations}

Wild type adult BALB/c mice were obtained from The Biotechnology Institute, the National Autonomous University of Mexico (UNAM) and housed under specific pathogen-free (SPF) conditions in the animal facilities (UPEAL) at the Center of Research and Advanced Studies (CINVESTAV-IPN). Trying to mimic the route and dosage as in natural DENV infection, mice were cutaneously infected with $6 \times 10^{4}$ PFU of DENV serotype 2 New Guinea reference strain in the shaved inguinal regions and boosted at day 7. UV-inactivated DENV (iDENV) and sterile endotoxin-free phosphate buffered saline (PBS) were used as experimental controls. The regional draining (inguinal) lymph nodes (DLNs) were obtained 3-, 7-, 14-, 16- or 23-days post-inoculation (p.i.). Experiments were performed in accordance with the national and institutional ethical guidelines for animal care and experimentation (approval number: 0070-13; CINVESTAV-IPN).

\section{DENV Stock Obtaining}

DENV stock was obtained in vitro by infecting the C6/36 cell line (from Aedes albopictus larvae) with brain extracts of infected neonate mice. C6/36 cells were grown in minimum essential medium eagle (MEM) supplemented with $10 \%$ Fetal Bovine Serum (Gibco, NY, USA), Amphotericin B, Penicillin, Streptomycin, Pyruvate, Vitamins and L-glutamine, at $34{ }^{\circ} \mathrm{C}$ in $75-\mathrm{cm}^{2}$ culture flask (Corning, NY, USA). Infection was performed when cells reached $95 \%$ of confluency. After $48 \mathrm{~h}$ of infection, culture supernatant containing DENV was collected and concentrated with Amicon Centrifugal Filter Units (Merk Millipore, MA, USA). Infectious virion quantification was performed using a plaque-forming assay in Monkey African Green kidney cell line (Vero) and reported as PlaqueForming Units (PFU)/mL. 


\section{Immunofluorescence Microscopy}

DLNs were obtained 7- and 14-days p.i., embedded in an optimal cutting temperature (OCT) compound Tissue Tek (Sakura FineTek, Torrance, CA, USA) and frozen in liquid nitrogen. $5 \mu \mathrm{m}$-slices of tissue were obtained with a Leica cryostat (Leica Microsystems) and put on Poly-L Lysine treated glass slides and fixed in cold acetone. Some slides were stain with Hematoxilin and Eosin (H\&E) following conventional histological protocols and others were rehydrated in PBS- $0.01 \%$ Tween-20, blocked with a casein solution (Power Block, BioGenex Laboratories, San Ramon, CA, USA) and labeled with the following primary antibodies in a PBS solution containing $1 \%(\mathrm{vol} / \mathrm{vol})$ of bovine serum albumin, $1 \%$ ( $\mathrm{vol} / \mathrm{vol})$ of normal human serum and $0.01 \%$ of sodium azide: Rat anti-mouse B220Brilliant Violet 450 from BioLegend (RA3-6B2; San Diego, CA, USA), Rat anti-mouse Thy 1.2-Biotin (53-2.1) and Rabbit anti-mouse Active Caspase-3-FITC (C92605.1) from BD Biosciences (San Jose, CA, USA), Rabbit anti-mouse Ki-67 (polyclonal) from Abcam (Cambridge, UK), Rat anti-mouse CD68 antibody (FA-11) from BioRad (Hercules, CA, USA) and Sheep anti-mouse IgD antiserum. Alexa Fluor 488-labelled anti-rabbit and anti-rat antibodies, Alexa Fluor 568-labelled anti-goat antibody and Alexa Fluor 555-labelled streptavidin were used as a secondary step and were incubated $1 \mathrm{~h}$ or $15 \mathrm{~min}$ at room temperature, respectively. DAPI (4',6-diamidino-2-phenylindole) was used for 5 min to stain nuclei. After 3 washings, slides were mounted in DABCO-Glycerol solution. In Situ Cell Death Detection Kit (Roche) was used for the TUNEL assay according the manufacturer instructions to detect apoptosis at single cell level. Images were captured with a Leica TCS SP8 AOBS Confocal microscopy using $\times 10$, $\times 40$ and $\times 100$ magnification objectives. Images were processed to obtain maximum-intensity projections (MIPs) and then assembled using the Auto-Align Layers tool in Photoshop to obtain the panoramic images of the whole DLNs. Quantification of areas and plot profiles of pixel intensity were obtained using ImageJ software (NIH).

\section{Flow Cytometry}

For lymphocyte analysis, single-cell suspensions were obtained by mechanical disaggregation of DLNs and passed through a $70 \mu \mathrm{m}$ cell strainer. Cell suspensions were blocked with a casein solution (Power Block, BioGenex Laboratories, San Ramón, CA, USA) to reduced nonspecific binding and then labelled with a mix of the following antibodies: Hamster monoclonal anti-CD3 (500A2), Rat monoclonal anti-CD4 (GK1.5), Rat monoclonal antiCD69 (H1.2F3), Rat monoclonal anti-B220 (RA3-6B2) from BD Biosciences (San Jose, CA, USA) and Rat monoclonal anti-CD8a (53-6.7) from eBioscience (San Diego, CA, USA) to detect membrane molecules. After a washing step with FACs buffer (PBS solution containing 1\% (vol/ vol) of fetal bovine serum and $0.01 \%$ of sodium azide), cells were treated with the Foxp3/Transcription Factor Fixation/Permeabilization Foxp3 kit (Invitrogen ${ }^{\mathrm{TM}}$ eBioscience $^{\mathrm{TM}}$ ) for intracellular detection of Ki-67 protein (rabbit polyclonal antibody), this last antibody was obtained from Abcam (Cambridge, UK). Appropriate fluorescent labelled streptavidin and anti-Rabbit secondary antibody were added to the cell suspensions. Samples were acquired in a BD LSR II Fortessa flow cytometer and data were analysed with the Flowjo Software (X.0.6 version for Windows, Ashland, OR, USA).

\section{Statistical Analysis}

Statistical analysis was performed using the GraphPad PrismSoftware (San Diego, CA, USA). Graphs show the data of the mean \pm the standard error of the mean (SEM). When the three experimental groups were compared (Naïve, iDENV and DENV), two-way analysis of variance (ANOVA) test was performed. A Bonferroni's multiple comparison test was applied and a $P$ value $<0.05$ was considered statistically significant. When two groups (Naïve and DENV) were compared, statistical significance was calculated using unpaired two-tailed Student's $t$ test.

\section{Results}

\section{After Cutaneous DENV Infection B Cell Follicles Expand and Host Large Clusters of Proliferating Cells While Few Scattered Proliferating T Cells Appear in DLN Paracortex}

Following cutaneous DENV infection in immunocompetent mice, an increase in the size of the DLNs and the formation of big GCs is induced, as previously reported (Yam-Puc et al. 2015). To evaluate more precisely the effects of cutaneous DENV inoculation upon the B and T cell compartments in situ, and the putative cell proliferation in each compartment, we probed for Ki-67 marker. We found that the B cell follicle area is increased 7 days after DENV infection, rising from $22.3 \% \pm 2.3 \%$ of the total DLN area in naïve DLNs to $29.7 \% \pm 0.3 \%$ in DLNs from infected animals. The B cell area of DENV-infected mice is hosting much more $\mathrm{Ki}-67+$ cells than naïve follicles do (Fig. 1A, 1B). The number of follicles that become Ki-67+ start to rise after 7 days of DENV immunization (Fig. 1C). While in the naïve follicles there were very few and small $\mathrm{Ki}-67+$ cell aggregates $(1.0 \% \pm 0.7 \%)$, at day 7 p.i. a big 


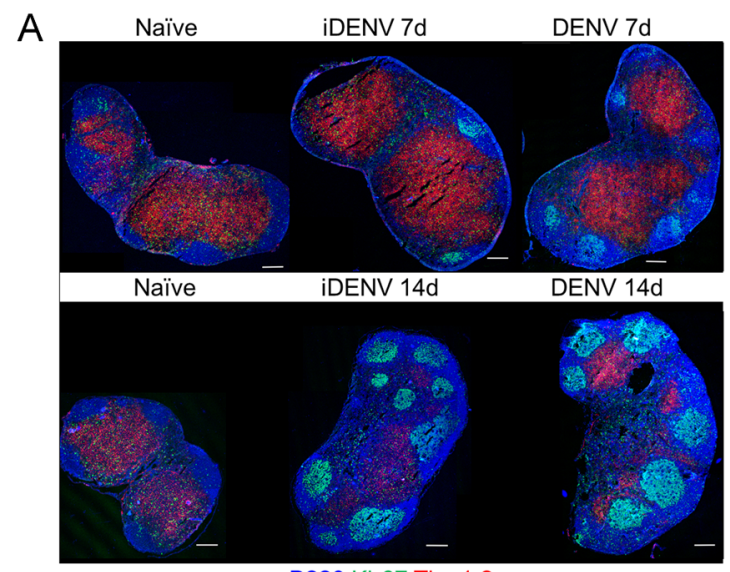

B220 Ki-67 Thy 1.2

B

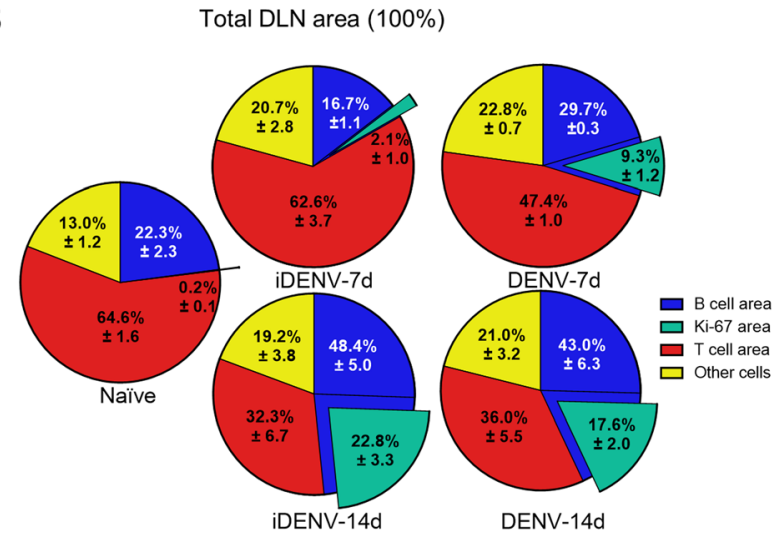

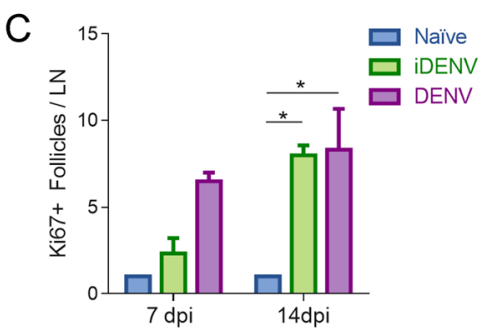

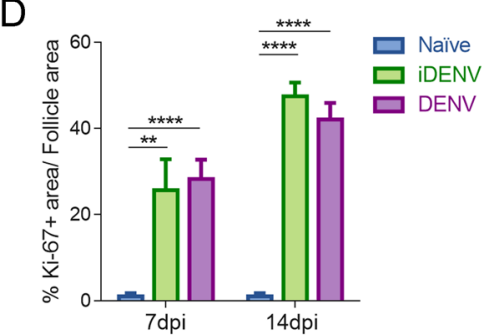

Fig. 1 Organization of $\mathrm{T}$ and $\mathrm{B}$ cell compartments in DLNs after cutaneous DENV infection. Mice were cutaneously infected with $6 \times 10^{4} \mathrm{PFU}$ of DENV and boosted at day 7 in each inguinal region. A Frozen DLN sections labelled for T lymphocytes (Thy 1.2, red), B lymphocytes (B220, blue) and a proliferation marker (Ki-67, green). The left panels show representative panoramic images of DLNs from non-infected mice (endotoxin-free PBS inoculated mice), whereas the middle and right panels show DLNs from iDENV- and DENVinoculated mice, respectively. Upper set depicts day 7 p.i. whereas

portion of the follicles was occupied by $\mathrm{Ki}-67+$ cells $(28.2 \% \pm 4.5 \%$, Fig. 1D). In contrast, the total $\mathrm{T}$ cell area (in the paracortex) seems slightly decreased in DENV-infected mice at 7 days p.i., from $64.6 \% \pm 1.6 \%$ to $47.4 \% \pm 1.0 \%$ (Fig. 1A, 1B). To evaluate the potential role of the sole presence of viral antigens upon lymphocyte activation and proliferation we inoculated mice with UVinactivated virus (iDENV), which contains all the viral componentes but is unable to replicate. We observed the DLNs of mice inoculated with iDENV behaved similar to the control non-infected group, but the Ki-67+ area started to increase at this time (day 7 p.i.). However, at 14 days post-DENV infection, the B cell area in DLNs is highly expanded, from $22.3 \% \pm 2.3 \%$ in non-infected conditions to $43.0 \% \pm 6.3 \%$ in infected animals, containing an increased number of proliferating $(\mathrm{Ki}-67+)$ follicles, with $\mathrm{Ki}-67+$ cells occupying up to $42.0 \% \pm 3.8 \%$ of the bottom set illustrates 14 days p.i. Scale bars $200 \mu \mathrm{m}$. B Pie charts show the proportion of $\mathrm{B}, \mathrm{T}, \mathrm{Ki}-67+$ and other cells from the total DLN area. Data in each slice represents the mean \pm SEM. C Numbers of $\mathrm{Ki}-67+$ follicles per DLN and D Ki-67+ area from the B cell follicles at 7- and 14-days p.i. are shown. Data are representative of two independent experiments with 3 mice in each experiment. Two way-ANOVA test followed by Bonferroni's test for multiple comparison between groups were performed, $* P<0.05$; $* * P<0.01$, and $* * * * P<0.0001$.

follicular area (Fig. 1A-1D). In contrast, the DLNs paracortical zone seems decreasing, from $64.6 \% \pm 1.6 \%$ to $36.0 \% \pm 5.5$ at 14 days p.i. A very similar pattern regarding both compartments was observed at this time point with the iDENV immunization (Fig. 1A-1D).

To analyse inside DLNs the detailed distribution of individual proliferating cells in the $\mathrm{T}$ and $\mathrm{B}$ cell compartments, we used a higher magnification. Ki-67+ cells inside follicles from DLNs of non-infected mice were very scant, and when small clusters were found, they contained (in average) less than 15 cells per follicle. In contrast, the innumerable $\mathrm{Ki}-67+$ proliferating cells inside B follicles of iDENV- and DENV-inoculated mice were very prominent at 14 days p.i. (Fig. 2A). We analysed individual images in two-dimensional graphs of pixel intensity along $300 \mu \mathrm{m}$ longitude and observed around 3-5 wide-shape peaks in iDENV- and DENV-inoculated mice, indicating a 

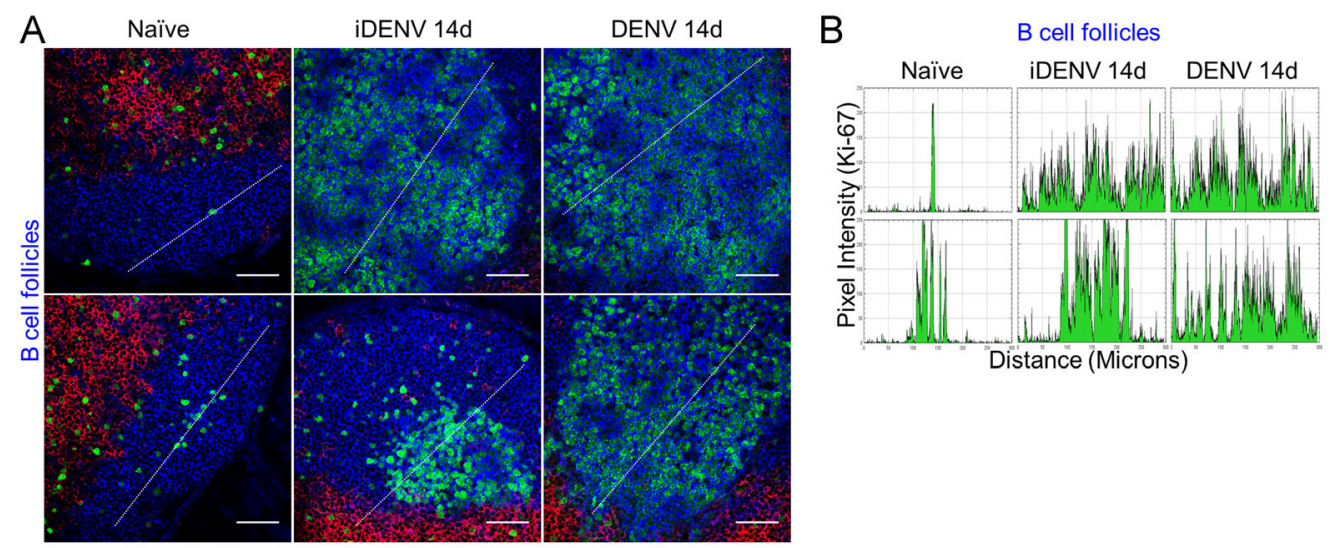

B220 Ki-67 Thy 1.2
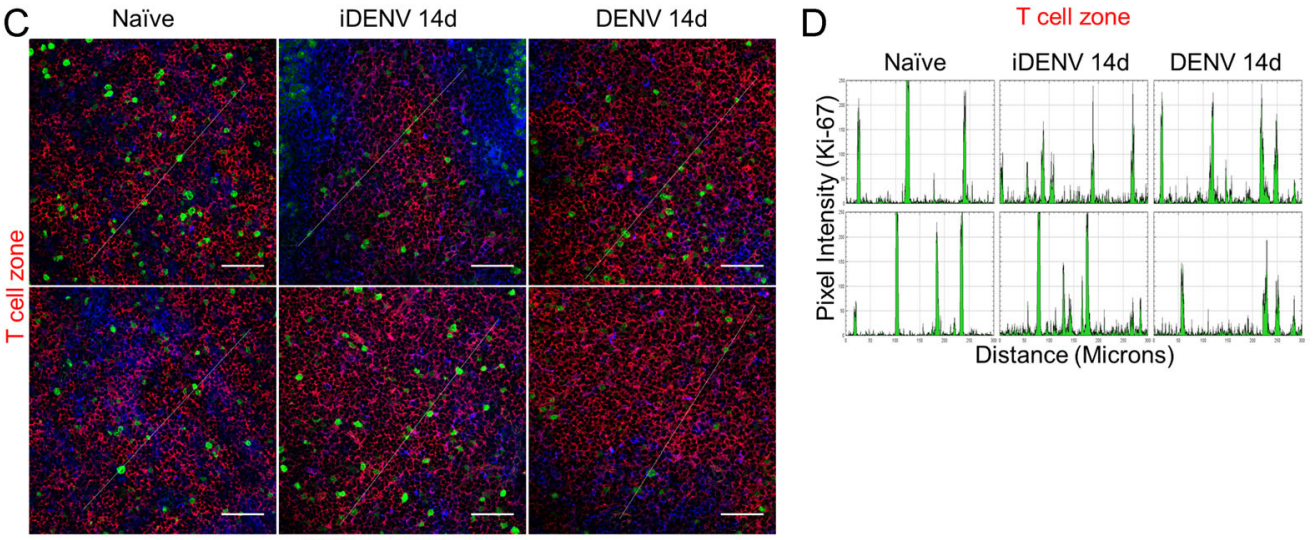

B220 Ki-67 Thy 1.2

Fig. 2 Distribution of proliferating cells in the B cell follicles and the paracortical area (T cells). Mice were infected as in Fig. 1. A Confocal microscopy images show B cells (B220, blue), T cells (Thy 1.2, red) and Ki-67 (green). Scale bars $50 \mu \mathrm{m}$. B Plot profiles generated from images in A, Y-axis represents pixel intensity and X-axis, $300 \mu \mathrm{m}$ distance (white lines in A). C Confocal microscopy images of paracortical/Thy $1.2+$ areas show scattered distribution of

clustering pattern of proliferating cells in this compartment (Fig. 2B). Of note are the dark areas observed in these profiles through the longitude analysed where the peaks decline, which correspond to TBMs containing apoptotic cells (see below). Concomitantly, we analysed paracortical areas where $\mathrm{T}$ lymphocytes and dendritic cells are found. It has been documented that after viral cutaneous infections with other virus such as herpes simplex virus, clusters of $\mathrm{CD} 8+\mathrm{T}$ and $\mathrm{CD} 4+\mathrm{T}$ cells are present in the paracortex (Hor et al. 2015). However, no clusters of proliferating $\mathrm{T}$ cells were seen in the paracortical areas of DLNs from DENV-infected mice (14 days p.i.). Instead, we found few scattered $\mathrm{Ki}-67+$ nuclei with an apparent random distribution, which displayed a very similar pattern with the PBS-inoculated mice (Fig. 2C). The iDENV and DENV plot profiles revealed single peaks of short longitude, indicative of single cells and each one with variable pixel intensity through the 300- $\mu \mathrm{m}$ longitude analysed (Fig. 2D). proliferating $(\mathrm{Ki}-67+)$ single cells after iDENV and DENV treatment, with a very similar pattern to non-immunized mice. Scale bars $50 \mu \mathrm{m}$. D iDENV and DENV plot profiles corresponding to the diagonal white lines in $\mathrm{C}$, show no different peak patterns compared to the control. Data are representative of two independent experiments performed in 3 mice each.

\section{Cutaneous DENV Inoculation is Ensued by Massive Recruitment of TBMs into the GCs}

TBMs represent a highly specialized subset inside GCs, these macrophages are crucial for the clearance of the B cells dying by apoptosis during the GC reaction (Flemming 1885). While assessing the GC reaction to cutaneous DENV infection we performed H\&E staining and noticed many condensed nuclei which at a higher magnification looked apparently "clustered" in defined separate groups, suggesting that several apoptotic bodies were probably phagocytosed by macrophages (Fig. 3A). We demonstrated by TUNEL assay many clusters of apoptotic cells inside the GCs (Fig. 3B). To corroborate that the phagocytic population involved were TBMs with apoptotic bodies inside the DENV-induced GCs, we double-labelled DLN sections using CD68 as a pan-macrophage marker and IgD to identify naïve B cell follicles. In DLNs from PBS- 

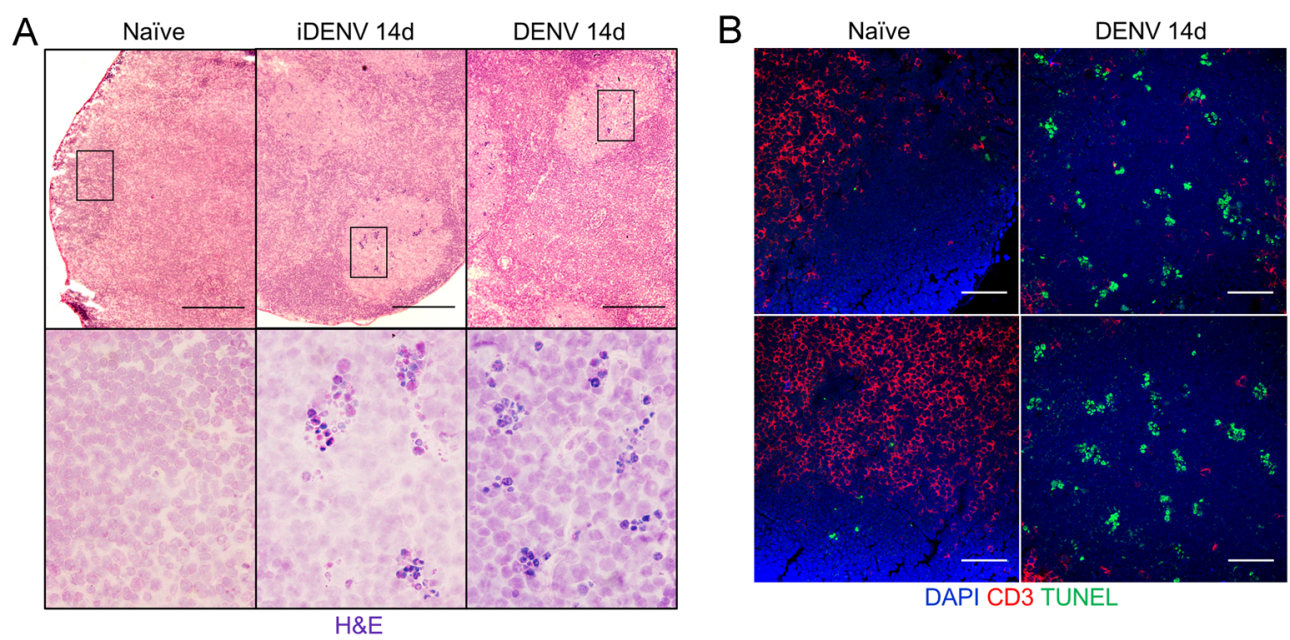

DAPI CD3 TUNEL
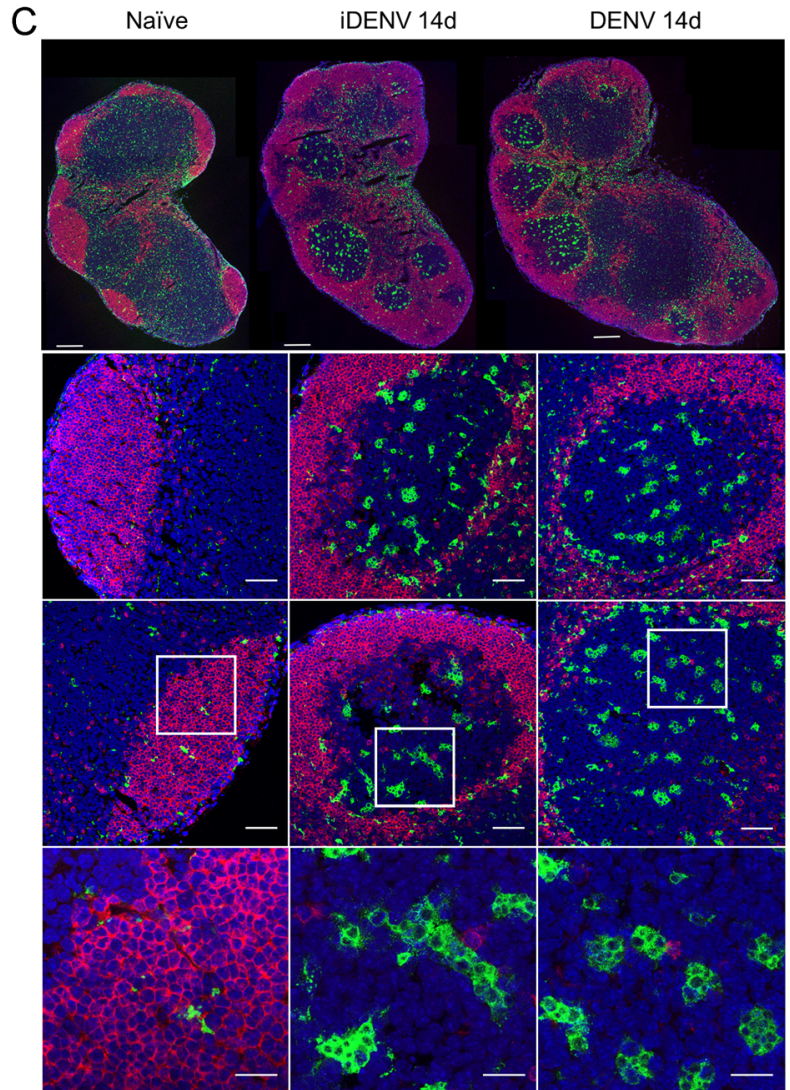

D

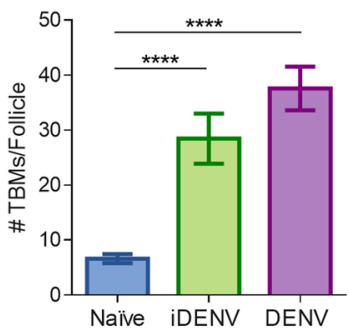

Fig. 3 Extensive recruitment of TBMs into GC follows cutaneous DENV infection. Mice were inoculated with either DENV, iDENV or PBS, as in Fig. 1 and analysed at day 14. A Cryosections of DLNs were stained with H\&E. Scale bars in upper panel $200 \mu \mathrm{m}$; magnifications of lower panel show black-boxed regions in upper panel, $60 \times$. B Confocal microscopy images show nuclei (DAPI, blue) and the distribution of separately grouped TUNEL + apoptotic cells (green) in the follicular areas (Thy 1.2-negative regions, red) after DENV immunization. Scale bars $50 \mu \mathrm{m}$. C Confocal microscopy images show DLNs with naïve B cells follicles (IgD- positive areas, red), macrophages (CD68+, green) and nuclei (DAPI, blue). The lower images represent higher magnifications of the area in white boxes. Scale bars in upper panel $200 \mu \mathrm{m}$, middle panels $50 \mu \mathrm{m}$ and lower panel $20 \mu \mathrm{m}$. D Bar charts show the mean \pm SEM of absolute numbers of TBMs inside each naïve follicle or DENV- and iDENV-induced GC. Images are representative of two independent experiments performed in 3 DLNs each. Statistical significance was calculated using ordinary one way-ANOVA test and Bonferroni's test for multiple comparison, $* * * * P<0.0001$. 
inoculated control mice, we found very few and small CD68+ macrophages inside non-reactive $(\mathrm{IgD}+) \mathrm{B}$ cell follicles. In these control animals the majority of the CD68+ cells were rather small macrophages and were widely scattered all along the medullar areas and the $\mathrm{T}$ cell zone (Fig. 3C). In contrast, in DLNs from iDENV- and DENV-immunized mice there were big, reactive, IgDnegative $\mathrm{B}$ cell follicles indicating an ongoing GC reaction. The distribution of big CD68+ macrophages was now remarkably confined into the GCs, while some were in the medullar areas and the $\mathrm{T}$ cell zones. This was more clearly seen at panoramic view at lower magnifications, suggesting a massive TBM recruitment into the GCs 14 days after DENV infection (Fig. 3C). Inside these large GCs, we found high numbers of big CD68+ TBMs representing $37.6 \pm 4.0$ per follicle in DENV- and $28.5 \pm 4.5$ in iDENV-inoculated mice, respectively, compared to only $6.6 \pm 0.8$ of them in control DLNs (Fig. 3D).

\section{Single-Cell Analysis of B and T Lymphocytes by Multicolor Flow Cytometry}

We quantified both the B cells and the CD4+ and CD8+ T lymphocyte proportions in DLN cell suspensions by multicolor flow cytometry. Consistent with the in situ analysis, we found increased percentages of B lymphocytes, from $23.06 \% \pm 0.9 \%$ in DLNs of non-infected mice to $40.5 \% \pm 3.1 \%$ in iDENV-immunized mice and $37.1 \% \pm 1.2 \%$ in DENV-infected mice (14 days p.i.,
Fig. 4A). We observed a similar pattern at 16 days p.i., and these proportions return to almost basal levels at 23 days p.i. (Fig. 4B). Although the percentage of total T cells is reduced at 14 and 16 days p.i., the proportion of individual CD4+ and CD8+ subsets among T lymphocytes only slightly changes with respect to the control, PBS-inoculated mice. Nevertheless, these latter changes show statistically significant differences compared with the control mice at 14 and 16 days p.i. (Fig. 4C).

\section{Differential Activation of B and T Cells During DENV Infection}

The responses of immune cells have been studied mostly in peripheral blood of DENV-infected patients, but this might not reflect the cellular reactions actually occurring inside the pertinent lymphoid organs (Green et al. 1999). We evaluated the expression of the CD69 activation marker in DLN lymphocytes of the three experimental groups. We observed that, compared to non-infected mice, both iDENV as well as DENV, elicited some expression of CD69 in the CD4+ $\mathrm{T}$ cells from 3 days after immunization (Fig. 5A). This profile of CD69 expression on CD4+ T cells is maintained until 23 days p.i. in DLNs from DENVinfected mice but not in iDENV-immunized mice (Fig. 5B). In comparison, the basal expression of CD69 on the CD8 + $\mathrm{T}$ cells showed some increase at 3- and 7-days p.i., but did not change at later times along the study (Fig. 5A, 5B). In contrast, after 3 days of DENV infection,

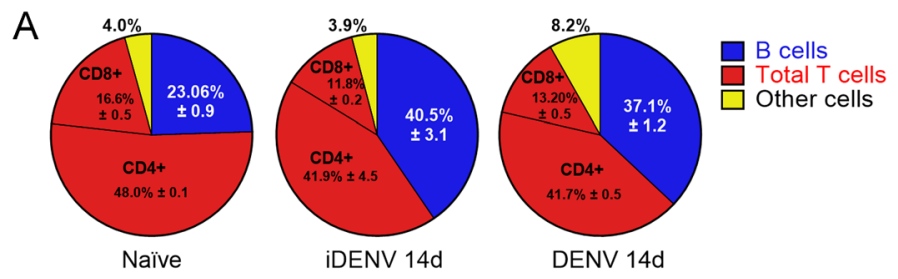

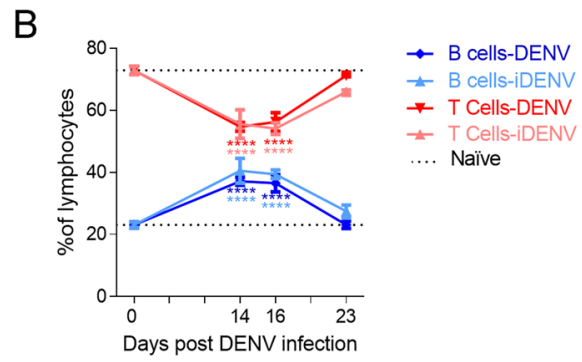

Fig. $4 \mathrm{~B}$ and $\mathrm{T}$ cell single-cell analysis by multiparametric flow cytometry. Mice were infected as in Fig. 1 and analysed at 14-, 16and 23- days p.i. Single cells suspensions were obtained from DLNs and labelled with monoclonal antibodies to B220, CD3, CD4 and CD8. A Pie charts show the mean \pm SEM of the proportion of each subpopulation from the total cells of DLNs at 14 days p.i. B The highest proportion of B lymphocytes is seen at 14 days p.i. while $\mathrm{CD} 3+$ cells decrease at the same time point. At 23 days p.i. the

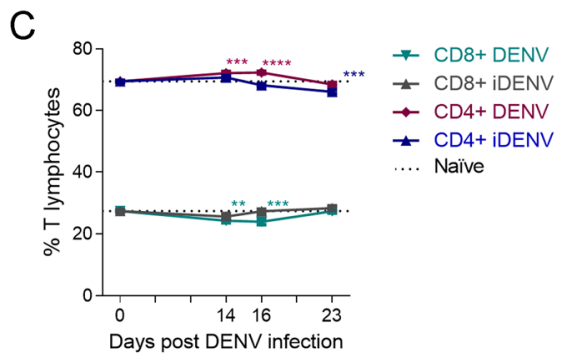

proportion of both populations returns to basal levels. C At 14 and 16 days p.i., the proportion of $\mathrm{CD} 4+\mathrm{T}$ lymphocytes increased very slightly compared to the controls, while the CD8+ population decreased after DENV treatment. Percentages are gated from CD3+ cells. Data are representative of two independent experiments from 4 mice each. Two way-ANOVA test followed by Bonferroni's test for multiple comparison between groups were performed, $* P<0.05$, $* * P<0.01, * * * P<0.001$ and $* * * * P<0.0001$. 
Fig. 5 Cutaneous DENV inoculation activates $B$ lymphocytes much more than $\mathrm{T}$ lymphocytes. DLN single-cell suspensions of control, iDENV and DENV infected mice were analysed by flow cytometry 3-, 7-, 14-, 16- and 23- days p.i. Labelling was performed with B220, CD3, CD4, CD8 and CD69 monoclonal antibodies. (A) Representative flow cytometry dot plots to show that CD4+ and CD8+ $\mathrm{T}$ cells slightly increase compared to the control, while B cells strongly upregulated CD69 at 3 days p.i. B Illustrates the percentages of activated (CD69+) lymphocytes among the $\mathrm{CD} 3+\mathrm{CD} 4+, \mathrm{CD} 3+$ $\mathrm{CD} 8+$ and the $\mathrm{B} 220+$ populations. C The percentage of change from basal levels in the expression of CD69 on B cells and T cells in iDENV- and DENV-immunized mice. Data are representative of two independent experiments from 4 mice each. Statistical significance was determined with two way-ANOVA test and Bonferroni's multiple comparison, $* P<0.05$, $* * P<0.01, * * * P<0.001$ and $* * * * P<0.0001$. the expression of CD69 on

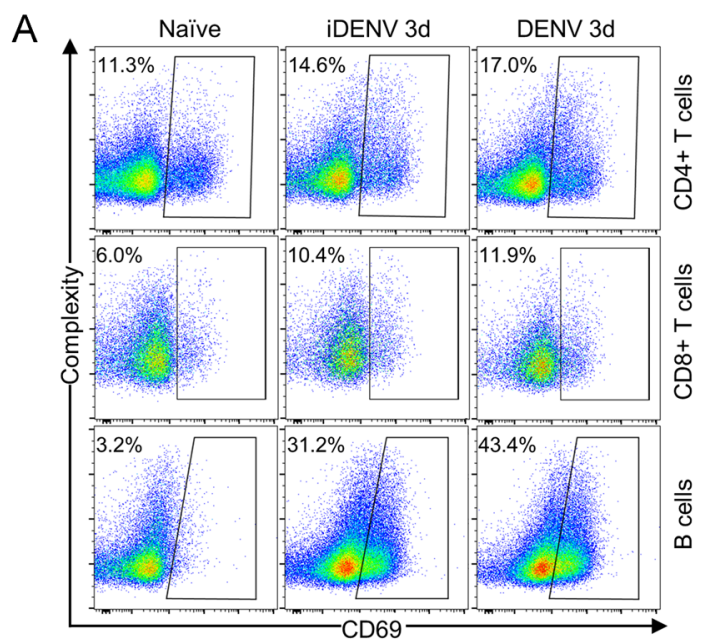

B

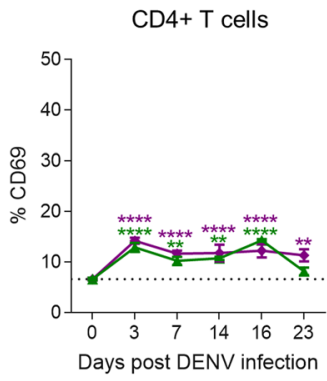

C

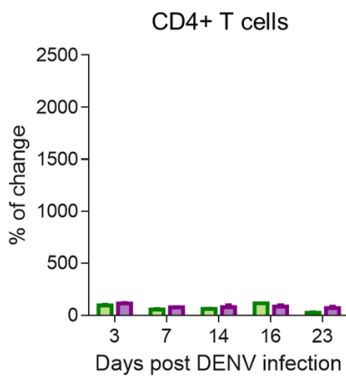

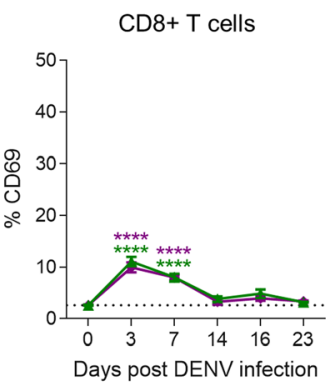

$\mathrm{CD} 8+\mathrm{T}$ cells

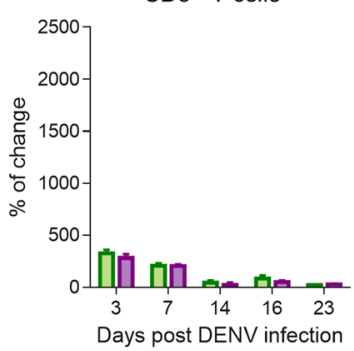

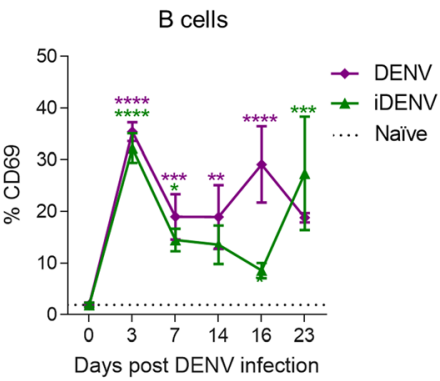

B cells

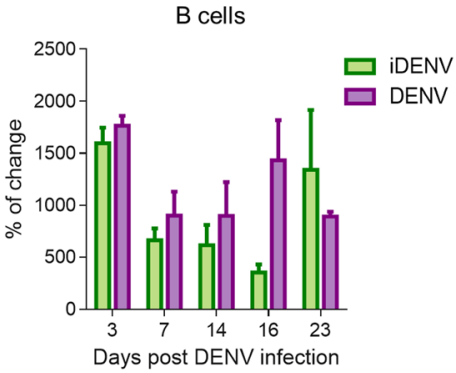

B cells showed an strong upregulation of CD69 decreasing at days 7 and 14 and increasing by 16 days p.i., with some decrease by day 23 p.i. (Fig. 5A, 5B). Thus, CD69+ B cells arose from very low levels, $1.9 \% \pm 0.4 \%$ in uninfected conditions, to reach the highest expresion at 3 days p.i. and with $35.4 \% \pm 1.8 \%$ and $29.0 \% \pm 7.3 \%$ at 16 days post DENV infection (Fig. 5A, 5B). This increase represents $1764.7 \%$ and $1431.9 \%$ of change from the basal expression at 3- and 16-days p.i., respectively. By comparison, $\mathrm{CD} 4+\mathrm{T}$ cells just show $84.3 \%$ of change compared to the naïve cells, whereas CD8+ T cells reach only $48.0 \%$ of change at 16 days p.i. (Fig. 5C).

\section{DLN B Cells But Not T Cells Show a Massive Proliferative Response to Cutaneous DENV Infection}

We wanted to measure the potential proliferative response of DLN lymphocytes to cutaneous DENV infection. We therefore assessed the nuclear expression of $\mathrm{Ki}-67$ protein by multicolor flow cytometry in B lymphocytes, and in CD4+ and CD8+ T cells (Fig. 6A, 6B). A very low basal percentage of $\mathrm{B}$ cells show proliferation in DLNs from non-infected mice, $2.27 \% \pm 0.3 \%$, but this percentage rises dramatically to $33.1 \% \pm 2.4 \%$ with DENV and to $25.3 \% \pm 3.2 \%$ with iDENV at day 16 p.i. (Fig. $6 \mathrm{~B}$ ). In the case of $\mathrm{T}$ lymphocytes, in CD4+ $\mathrm{T}$ cells we did not find even a minor proliferative reaction to DENV, with $3.1 \% \pm 0.4 \%$ in DENV-infected mice compared to 

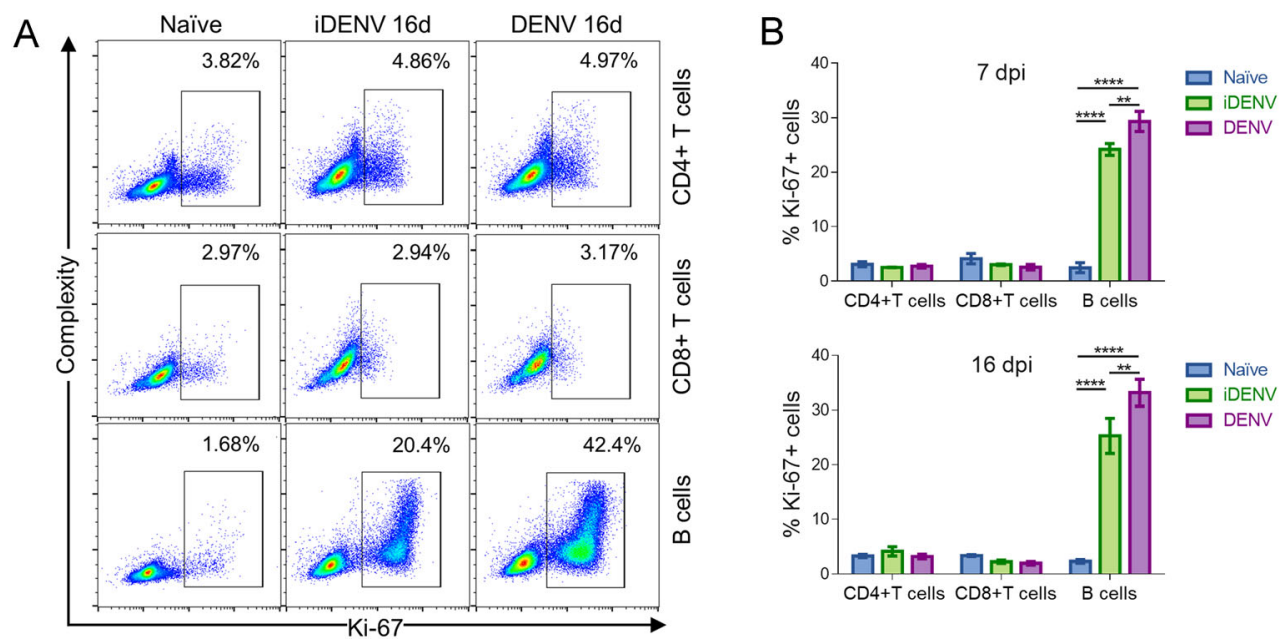

Fig. 6 DENV induces strong proliferation on B lymphocytes but not on $\mathrm{T}$ cells. DLN cell suspensions were obtained 7- and 16-days post cutaneous inoculation of DENV, iDENV and endotoxin-free PBS and labelled as in Fig. 4. Nuclear permeabilization was performed for detection of Ki-67 protein. A Representative flow cytometry dot plots show the appearance of an abundant $\mathrm{Ki}-67+$ population in $\mathrm{B} 220+\mathrm{B}$ cells but not in CD4+ nor in CD8+ T cells after iDENV and DENV inoculation. B Bar graphs represent the mean \pm SEM of the percentage of $\mathrm{Ki}-67+$ cells showing a massive $\mathrm{B}$, but not $\mathrm{T}$ cell proliferation, both at 7- and 16-days p.i. Two independent experiments from 4 mice each were pooled and graphed. Statistical significance was calculated using two way-ANOVA test and Bonferroni's test for multiple comparison, $* * P<0.01$ and $* * * * P<0.0001$.

were found in the paracortex of DLNs from DENV-infected mice. In both cases, the percentages lacked statistically significant differences, compared with the noninfected mice (Fig. 7C).

\section{Discussion}

Since one of the crucial risk factors to develop severe dengue is the prior immune response to DENV and considering that $\mathrm{T}$ and $\mathrm{B}$ lymphocytes encompass the specificity and memory of adaptative immune responses, it is conceivable that they could be involved in DENV immunopathology (Burke et al. 1988; Graham et al. 1999; Endy et al. 2002). However, their potential protective versus pathological role in the disease is not fully understood, especially for $\mathrm{T}$ lymphocytes (Malavige and $\mathrm{Ogg}$ 2013; Screaton et al. 2015; Rivino 2016). This, in part might be due to the fact that DENV specific T cells represent a very low proportion of circulating cells during the acute phase (median $0.75 \%$, mean $2.3 \%$ ) (Friberg et al. 2011); no response, up to $0.1 \%$ and $2.5 \%$ (Mongkolsapaya et al. 2006); 0.02\%-2.5\% (Mongkolsapaya et al. 2003), when the severe symptoms start. Rather, a differentiated form of B cells, the plasmablasts, have been found as high as a 1000 -fold increase, constituting up to $30 \%$ of the total PBMCs, in some cases even mimicking a plasma cell leukaemia during acute DENV infection (Gawoski and Ooi 2003; Balakrishnan et al. 2011; Wrammert et al. 2012). Most of these plasmablasts (70\%) recognize DENV (Fig. 7B). Likewise, scarce TUNEL+ apoptotic T cells 

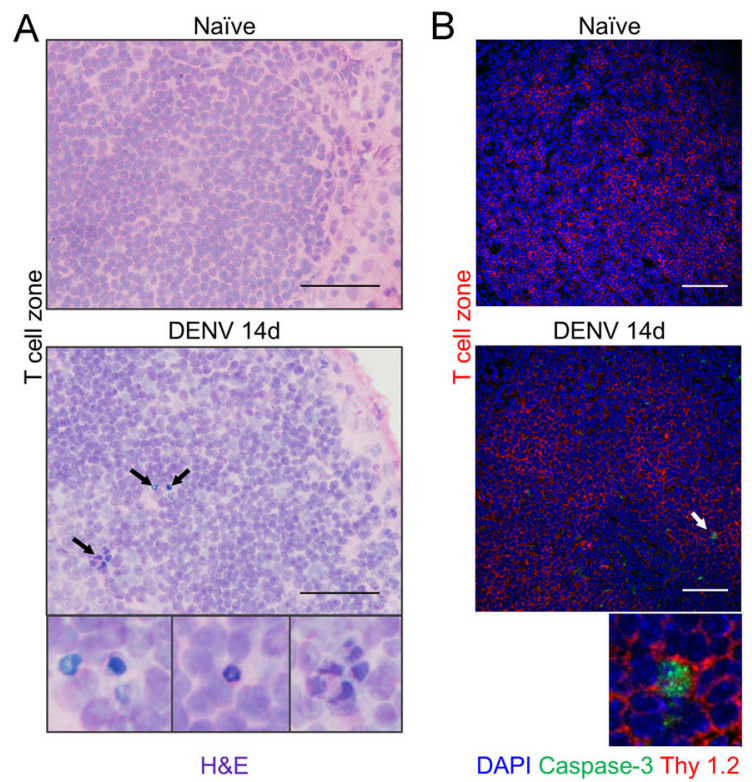

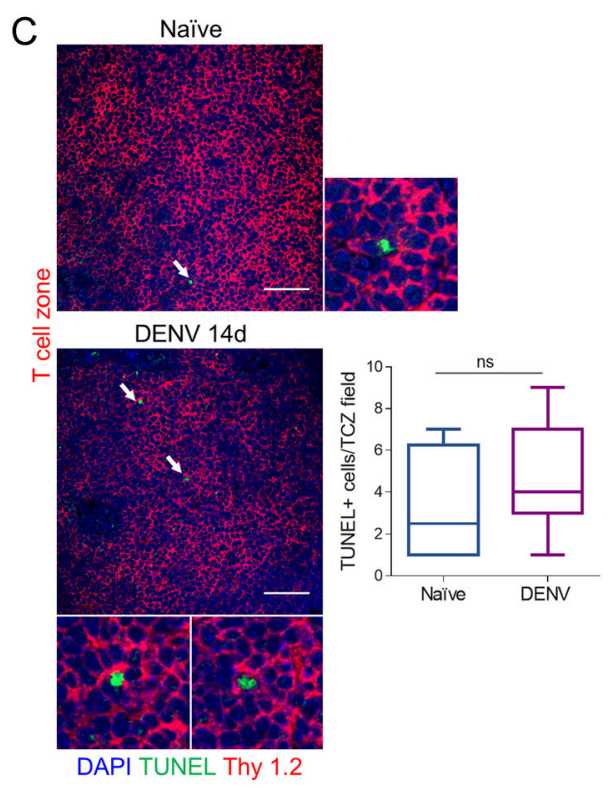

Fig. $7 \mathrm{~T}$ cells do not seem to undergo significant apoptosis after DENV inoculation. Regional DLNs were frozen after the corresponding treatments. A DLN cryosections were stained with H\&E. Black arrows in the images as well as the magnifications below indicate apoptotic bodies. Scale bars $50 \mu \mathrm{m}$. B Immunofluorescence in tissue sections to detect active caspase-3 (green) in the paracortical area (Thy $1.2+$, red). Nuclei were stained with DAPI (blue). Magnification below show caspase $3+\mathrm{T}$ cells in DENV-infected

(Wrammert et al. 2012) and are serotype cross-reactive (Garcia-Bates et al. 2013), thus revealing the important involvement of B cells.

Most of DENV research has been performed in circulating blood cells from infected patients, in our study we evaluated the corresponding lymph nodes (DLNs) as crucial lymphoid organs evolved to facilitate the potential encounters between antigens and the low frequency of antigen-specific lymphocytes (Flajnik 2018). Using a previously established model of cutaneous DENV infection in immunocompetent mice (Yam-Puc et al. 2015), we found by in situ evaluation and single-cell analysis, a nearly absent $\mathrm{T}$ cell proliferative response to DENV infection (measured by the expression of Ki-67), in both the CD4+ and the CD8 $+\mathrm{T}$ cells. In line with this, a lack of $\mathrm{T}$ cells expansion, an impaired proliferation, defects on IFN- $\gamma$ production, and suboptimal $\mathrm{T}$ cell degranulation in the acute phase have been documented in patients, some reports attributed the poor $\mathrm{T}$ cell response to apoptosis (Mongkolsapaya et al. 2003, 2006; Dung et al. 2010). To explore this last possibility in our model, we assessed cell death in DLNs by three different approaches but we did not find a significant increase in $\mathrm{T}$ cell apoptosis ratio under DENV compared with non-infected controls, suggesting that apoptosis might not be the major determinant for $\mathrm{T}$ cell unresponsiveness. tissue. C White arrows indicate T cells (Thy 1.2+, red fluorescence) with fragmented DNA (TUNEL+, green fluorescence) in situ. Scale bars $50 \mu \mathrm{m}$. Floating bar charts show the mean \pm SEM of the numbers of apoptotic cells in the T cell zone (TCZ) after DENV infection and those in the PBS-control DLNs. Data are representative of two independent experiments from 4 mice each. Statistical significance was calculated using unpaired two-tailed Student's $t$ test. $n s$ not significantly different.

According to transcriptomic analysis, downregulation of TCR signalling genes is elicited by DENV impairing the T cell production of INF- $\gamma$ and the CD69 upregulation in a TCR-dependent manner (Chandele et al. 2016), a fact that might be relevant to the minor $\mathrm{T}$ cell responses that we found, as we did not observe substantially increased CD69 expression in T cells. Other authors have shown decreased $\mathrm{T}$ cell proliferation in vitro due to defects in the accessory cell population in PBMCs (during acute dengue illness), seemingly related to low supply of IL-2 and IL-7 cytokines (Mathew et al. 1999). Dendritic cells as targets for DENV infection (Wu et al. 2000) and replication might be impaired to prime $\mathrm{T}$ cells. This has been confirmed in vitro, but only in certain DC subsets or when using high load of viral MOIs (Nightingale et al. 2008; Cerny et al. 2014).

There are other possibilities for the nearly absent $\mathrm{T}$ cell responses, for instance lack of antigen availability in vivo, but we have previously found the presence of DENV in dendritic cells in DLN paracortex soon after fluorescently labelled-DENV inoculation via skin (Marcial-Juárez et al. 2017). Another possibility is the upregulation of inhibitory molecules such as PD-1, LAG-3 and CTLA-4, indeed they have been found increased in $\mathrm{T}$ cells during DENV infection (Chandele et al. 2016). On the other hand, costimulatory molecules in dendritic cells have been found diminished after in vitro infection (Nightingale et al. 2008), 
all these events could impact an optimal $\mathrm{T}$ cell priming by antigen presenting cells (APCs). As we did not observe any increase in the proliferation nor in the activation of CD4+ and CD8+ T cells after iDENV and DENV inoculation, and since $\mathrm{T}$ lymphocytes are not a direct target for DENV (Blackley et al. 2007), it could be that DENV replication is not directly triggering this $\mathrm{T}$ cell unresponsiveness.

Certain lymphocyte activation markers have been determined either in PBMCs of dengue patients or after in vitro stimulation with DENV, some are even considered as a hallmark of severe cases, such as CD69 (Green et al. 1999; Correa et al. 2015; Chandele et al. 2016). We found a prominent increased expression of CD69 in B cells mainly at 3-days and until 23-days after cutaneous DENV infection. In contrast, $\mathrm{T}$ cells showed a very minor expression of CD69 compared to basal conditions, and it was much lower when compared to CD69 upregulation by B cells. Downregulation of CCR7 on T cells has been reported (Chandele et al. 2016) and together with the low expression of CD69 that we observed, this could indicate that $\mathrm{T}$ cells might not be retained inside DLNs, since is reported that CD69 participates in preventing lymphocytes leaving the lymph nodes (Cyster and Schwab 2012).

Remarkably, we found a large increase in the area occupied by proliferating B cells as well as in the number of Ki-67+ clusters inside DLN follicles. Since the proportion of B cells in the blood of DENV patients is just mild or not substantially increased, there is the possibility of B cells being retained in lymphoid organs (Jampangern et al. 2007; Garcia-Bates et al. 2013; Nivarthi et al. 2019). The idea of B cell proliferation in lymphoid organs during the natural infection is supported by the fact that numerous plasmablasts are found in the circulation of DENV-infected patients (Wrammert et al. 2012; Garcia-Bates et al. 2013). In line with the lymphocyte retention theory, we found CD69 strongly expressed in DLN B cells. CD69 blocks the egress of lymphocytes from lymph nodes, presumably allowing more time for further differentiation (Cyster and Schwab 2012).

The great proportion of $\mathrm{B}$ cells undergoing activation and proliferation in lymph nodes supports the idea of a polyclonal, BCR-independent activation, like other viruses reportedly do (Jellison et al. 2007). The fact that B cells bear and respond via innate receptors such as TLRs [for instance, a TLR-7 agonist plus IL-2 and IL-6 induce proliferation in naïve B cells and production of $\operatorname{IgM}$ and $\operatorname{IgG}$ in vitro (Glaum et al. 2009)] supports the notion of polyclonal activation, perhaps at early stages. In fact, polyclonal B cell activation induced by DENV and evidenced by production of IgM and IL-6 has been demonstrated in vitro, apparently mediated by CD81 (a crosslinker of CD19 and BCR) (Correa et al. 2015). DENV antigens inside follicles in human (post mortem) lymphoid tissue have been reported (Aye et al. 2014), and we have tracked fluorescent-DENV in DLNs and found it inside B cell follicles $1 \mathrm{~h}$ upon skin inoculation (Marcial-Juárez et al. 2017), indicative that DENV particles are indeed available to $\mathrm{B}$ cells.

In another study, the low proportion of somatic hypermutated antibody genes observed in humans during the acute phase of DENV infection, led authors to propose that $\mathrm{B}$ cells are undergoing an "innate-like" activation and extrafollicular reactions early during the primary infection (Godoy-Lozano et al. 2016). As we previously reported big GCs in DLNs after cutaneous DENV infection, plus the high rate of proliferation inside $B$ cell follicles, made us to consider the possibility that $\mathrm{B}$ cell responses or maturation via the $\mathrm{GC}$ reaction could be altered. It is worth mentioning that the classical compartmentalization of GCs in light zone and dark zone was absent in our studies, apparently due to the large expansion of proliferating $B$ cells inside follicles upon DENV infection. Of note, along with the extensive B cell proliferation, we found a high number of CD68+ TBMs and many dead cells, but exclusively in the B cell proliferating area (dark zone). This was detected by histology and confirmed by TUNEL. Normally, most TBMs (75\%) would appear in the light zone (Hardie et al. 1993; MacLennan 1994). In fact, TBMs have been reported in human lymphoid tissue from fatality cases due to DENV infection (Aye et al. 2014).

In summary, we found an early and strong B cell activation and proliferation in DLNs which is accompanied by an impressive recruitment of TBMs inside GCs after cutaneous DENV infection, this, and the very poor $\mathrm{T}$ cell responses observed correlate to some extent with the findings in naturally infected humans. We believe that these observations contribute to better understand the fundamental immune response triggered by DENV. More detailed studies not only on B cell differentiation through the study of the GC microanatomy, but also of the affinity maturation, selection and the production of protective and non-neutralizing antibodies and memory cells, are all of great interest and suitable to perform in this in vivo model. Likewise, the putative role of $\mathrm{T}$ cells in this important viral infection can be assessed simultaneously.

Acknowledgements We thank Professor Leopoldo Flores-Romo to share his passion for the study of the immune responses during early life and against pathogens. He will be greatly missed among his students and the Mexican community of immunologists. His legacy will last forever. Edith Marcial-Juárez and Raúl Antonio MaquedaAlfaro held a scholarship from The National Council for Science and Technology of Mexico, CONACYT. Leopoldo Flores-Romo, Leticia Cedillo-Barrón and Julio Garcia-Cordero are members of the National System of Researchers, SNI- CONACYT. The authors thank the help from Jaime Escobar at the Confocal Microscopy facilities, from Victor Rosales at the FACS facilities and Yolanda Sánchez-Chávez for the excellent technical assistance provided. This work was 
supported by a grant from CONACYT-Mexico (221102) to Leopoldo Flores-Romo and by a CINVESTAV grant to Leticia Cedillo-Barrón.

Author Contributions EM-J, LC-B, and LF-R conceived and designed the experiments. EM-J, JG-C, RAM-A and RES-L performed the experiments and the acquisition of data. EM-J, LC-B, LES-T and LF-R analysed the data, interpretation, and discussion. EM-J and LF-R wrote and revised the paper. LF-R finalized the manuscript.

\section{Compliance with Ethical Standards}

Conflict of interests The authors declare that they have no conflict of interest.

Animal and Human Rights Statement Experiments were performed in accordance with the national (NOM-062-ZOO-1999) and institutional (CICUAL approval number: 0070-13) ethical guidelines for animal care and experimentation (CINVESTAV-IPN), Mexico.

\section{References}

Arkin F (2019) Dengue researcher faces charges in vaccine fiasco. Science $364: 320$

Aye KS, Charngkaew K, Win N et al (2014) Pathologic highlights of dengue hemorrhagic fever in 13 autopsy cases from Myanmar. Hum Pathol 45:1221-1233

Balakrishnan T, Bela-Ong DB, Toh YX et al (2011) Dengue virus activates polyreactive, natural $\mathrm{IgG} \mathrm{B}$ cells after primary and secondary infection. PLoS ONE 6:e29430

Bhatt S, Gething PW, Brady OJ et al (2013) The global distribution and burden of dengue. Nature 496:504-507

Blackley S, Kou Z, Chen H et al (2007) Primary human splenic macrophages, but not $\mathrm{T}$ or B cells, are the principal target cells for dengue virus infection in vitro. J Virol 81:13325-13334

Buddhari D, Aldstadt J, Endy TP et al (2014) Dengue virus neutralizing antibody levels associated with protection from infection in Thai Cluster studies. PLoS Negl Trop Dis 8:1-10

Burke DS, Scott RM, Johnson DE, Nisalak A (1988) A prospective study of dengue infections in Bangkok. Am J Trop Med Hyg $38: 172-180$

Cannon MJ, Stott EJ, Taylor G, Askonas BA (1987) Clearance of persistent respiratory syncytial virus infections in immunodeficient mice following transfer of primed $\mathrm{T}$ cells. Immunology 62:133-138

Cerny D, Haniffa M, Shin A et al (2014) Selective susceptibility of human skin antigen presenting cells to productive dengue virus infection. PLoS Pathog 10:e1004548

Chandele A, Sewatanon J, Gunisetty S et al (2016) Characterization of human CD8 T cell responses in dengue virus-infected patients from India. J Virol 90:11259-11278

Channappanavar R, Fett C, Zhao J et al (2014) Virus-specific memory CD8 $\mathrm{T}$ cells provide substantial protection from lethal severe acute respiratory syndrome coronavirus infection. J Virol 88:11034-11044

Chatterjee P (2019) Dengvaxia researcher charged. Lancet Infect Dis 19:584-585

Correa ARV, Berbel ACER, Papa MP et al (2015) Dengue virus directly stimulates Polyclonal B cell activation. PLoS ONE 10:1-20

Cyster JG, Schwab SR (2012) Sphingosine-1-phosphate and lymphocyte egress from lymphoid organs. Annu Rev Immunol 30:69-94
Dick OB, San Martín JL, Montoya RH et al (2012) Review: the history of dengue outbreaks in the Americas. Am J Trop Med Hyg 87:584-593

Dung NTP, Le DH, Van TN et al (2010) Timing of CD8+ T cell responses in relation to commencement of capillary leakage in children with dengue. J Immunol 184:7281-7287

Endy TP, Chunsuttiwat S, Nisalak A et al (2002) Epidemiology of inapparent and symptomatic acute dengue virus infection: a prospective study of primary school children in Kamphaeng Phet, Thailand. Am J Epidemiol 156:40-51

Flajnik MF (2018) A cold-blooded view of adaptive immunity. Nat Rev Immunol 18:438-453

Flemming W (1885) Studien über Regeneration der Gewebe. Arch mikr Anat 24:50-91

Friberg H, Bashyam H, Toyosaki-Maeda T et al (2011) Crossreactivity and expansion of dengue-specific $\mathrm{T}$ cells during acute primary and secondary infections in humans. Sci Rep 1:1-9

Garcia-Bates TM, Cordeiro MT, Nascimento EJM et al (2013) Association between magnitude of the virus-specific plasmablast response and disease severity in dengue patients. J Immunol 190:80-87

Gawoski JM, Ooi WW (2003) Dengue fever mimicking plasma cell leukemia. Arch Pathol Lab Med 127:1026-1027

Glaum MC, Narula S, Song D et al (2009) Toll-like receptor 7-induced naive human B-cell differentiation and immunoglobulin production. J Allergy Clin Immunol 123:224.e4-230.e4

Godoy-Lozano EE, Téllez-Sosa J, Sánchez-González G et al (2016) Lower IgG somatic hypermutation rates during acute dengue virus infection is compatible with a germinal center-independent B cell response. Genome Med 8:1-19

Graham RR, Juffrie M, Tan R et al (1999) A prospective seroepidemiologic study on dengue in children four to nine years of age in Yogyakarta, Indonesia I. Studies in 1995-1996. Am J Trop Med Hyg 61:412-419

Green S, Pichyangkul S, Vaughn DW et al (1999) Early CD69 expression on peripheral blood lymphocytes from children with dengue hemorrhagic fever. J Infect Dis 180:1429-1435

Halstead SB (1970) Observations related to pathogensis of dengue hemorrhagic fever. VI. Hypotheses and discussion. Yale J Biol Med 42:350-362

Halstead SB, O'Rourke EJ (1977) Antibody-enhanced dengue virus infection in primate leukocytes. Nature 265:739-741

Hardie DL, Johnson GD, Khan M, MacLennan ICM (1993) Quantitative analysis of molecules which distinguish functional compartments within germinal centers. Eur $\mathrm{J}$ Immunol 23:997-1004

Hor JL, Whitney PG, Zaid A et al (2015) spatiotemporally distinct interactions with dendritic cell subsets facilitates CD4+ and $\mathrm{CD} 8+\mathrm{T}$ cell activation to localized viral infection. Immunity 43:554-565

Jampangern W, Vongthoung K, Jittmittraphap A et al (2007) Characterization of atypical lymphocytes and immunophenotypes of lymphocytes in patients with dengue virus infection. Asian Pac J Allergy Immunol 25:27-36

Jellison ER, Guay HM, Szomolanyi-Tsuda E, Welsh RM (2007) Dynamics and magnitude of virus-induced polyclonal B cell activation mediated by BCR-independent presentation of viral antigen. Eur J Immunol 37:119-128

MacLennan IC (1994) Germinal centers. Annu Rev Immunol 12:117-139

Malavige GN, Ogg GS (2013) T cell responses in dengue viral infections. J Clin Virol 58:605-611

Marcial-Juárez E, Yam-Puc JC, Cedillo-Barrón L et al (2017) Travelling with dengue: from the skin to the nodes. In: Aparecida Sperança M (ed) Dengue: immunopathology and 
control strategies, 1st edn. IntechOpen Limited, London, pp 27-42

Mathew A, Kurane I, Green S et al (1999) Impaired T cell proliferation in acute dengue infection. $\mathrm{J}$ Immunol 162:5609-5615

Mongkolsapaya J, Dejnirattisai W, Xu X et al (2003) Original antigenic $\sin$ and apoptosis in the pathogenesis of dengue hemorrhagic fever. Nat Med 9:921-927

Mongkolsapaya J, Duangchinda T, Dejnirattisai W et al (2006) T cell responses in dengue hemorrhagic fever: are cross-reactive $\mathrm{T}$ cells suboptimal? J Immunol 176:3821-3829

Ndhlovu ZM, Kamya P, Mewalal N et al (2015) Magnitude and kinetics of $\mathrm{CD} 8+\mathrm{T}$ cell activation during hyperacute HIV infection impact viral set point. Immunity 43:591-604

Nightingale ZD, Patkar C, Rothman AL (2008) Viral replication and paracrine effects result in distinct, functional responses of dendritic cells following infection with dengue 2 virus. J Leukoc Biol 84:1028-1038

Nivarthi UK, Tu HA, Delacruz MJ et al (2019) Longitudinal analysis of acute and convalescent $\mathrm{B}$ cell responses in a human primary dengue serotype 2 infection model. EBioMedicine 41:465-478

Rivino L (2016) T cell immunity to dengue virus and implications for vaccine design. Expert Rev Vaccines 15:443-453

Rothman AL (2004) Dengue: defining protective versus pathologic immunity. J Clin Invest 113:946-951

Rush B (1789) An account of the bilious remitting fever, as it appeared in Philadelphia in the summer and autumn of the year 1780, Medical In. Prichard and Hall, Philadelphia

Sabin AB (1952) Research on Dengue during World War II 1. Am J Trop Med Hyg 1:30-50
Sangkawibha N, Rojanasuphot S, Ahandrik S et al (1984) Risk factors in dengue shock syndrome: a prospective epidemiologic study in Rayong, Thailand: I. The 1980 outbreak. Am J Epidemiol 120:653-669

Screaton G, Mongkolsapaya J, Yacoub S, Roberts C (2015) New insights into the immunopathology and control of dengue virus infection. Nat Rev Immunol 15:745-759

Slütter B, Pewe LL, Kaech SM, Harty JT (2013) Lung airwaysurveilling CXCR3hi Memory CD8+ $\mathrm{T}$ cells are critical for protection against influenza A virus. Immunity 39:939-948

Taylor PM, Askonas BA (1986) Influenza nucleoprotein-specific cytotoxic $\mathrm{T}$-cell clones are protective in vivo. Immunology $58: 417-420$

WHO (2012) Global strategy for dengue prevention and control 2012-2020. WHO Press, Geneva

WHO (2019) Dengue and severe dengue. In: WHO. https://www.who. $\mathrm{int} /$ news-room/fact-sheets/detail/dengue-and-severe-dengue. Accessed 30 Sep 2019

Wrammert J, Onlamoon N, Akondy RS et al (2012) Rapid and massive virus-specific plasmablast responses during acute dengue virus infection in humans. J Virol 86:2911-2918

Wu S-JJ, Grouard-Vogel G, Sun W et al (2000) Human skin Langerhans cells are targets of dengue virus infection. Nat Med 6:816-820

Yam-Puc JC, García-Cordero J, Calderón-Amador J et al (2015) Germinal center reaction following cutaneous dengue virus infection in immune-competent mice. Front Immunol 6:1-9

Yap KL, Ada GL, McKenzie IFC (1978) Transfer of specific cytotoxic T lymphocytes protects mice inoculated with influenza virus. Nature 273:238-239 